\title{
RICCI FLOW AND THE POINCARÉ CONJECTURE
}

\author{
SIDDHARTHA GADGIL AND HARISH SESHADRI
}

The field of Topology was born out of the realisation that in some fundamental sense, a sphere and an ellipsoid resemble each other but differ from a torus - the surface of a rubber tube (or a doughnut). A striking instance of this can be seen by imagining water flowing smoothly on these. On the surface of a sphere or an ellipsoid (or an egg), the water must (at any given instant of time) be stationary somewhere. This is not so in the case of the torus.

In topology, we regard the sphere and the ellipsoid as having the same topological type, which we make precise later. Topology is the study of properties that are shared by objects of the same topological type. These are generally the global properties. Understanding the different topological types of spaces, the so called classification problem, is thus a fundamental question in topology.

In the case of surfaces (more precisely closed surfaces), there are two infinite sequences of topological types. The first sequence, consisting of the so called orientable surfaces, consist of the sphere, the torus, the 2-holed torus, the 3-holed torus and so on (see figure 1). One would like to have a similar classification in all dimensions. However, due to fundamental algorithmic issues, it is impossible to have such a list in dimensions four and above.

There is a simple way to characterise the sphere among surfaces. If we take any curve on the sphere, we can shrink it to a point while remaining on the sphere. A space with this property is called simply-connected. A torus is not simply-connected as a curve that goes around the torus cannot be shrunk to a point while remaining on the torus. In fact, the sphere is the only simply-connected surface.

In 1904, Poincaré raised the question as to whether a similar characterisation of the (3-dimensional) sphere holds in dimension 3. That this is so has come to be known as the Poincaré conjecture. As topology exploded in the twentieth century, several attempts were made to prove this (and some to disprove it). However, at the turn of the millennium this remained unsolved. Surprisingly, the higher dimensional analogue of this statement turned out to be easier and has been solved.

In 2002-2003, three preprints ([8], [9] and [10]) rich in ideas but frugal with details, were posted by the Russian mathematician Grisha Perelman, who had been working on this in in solitude for seven years at the Steklov Institute. These were based on the Ricci flow, which was introduced by Richard Hamilton in 1982. Hamilton had developed the theory of Ricci flow through the 1980's and 1990's, proving many important results and developing a programme [4] which, if completed, would lead to the Poincaré conjecture and much more. Perelman introduced a series of highly original ideas and powerful techniques to complete Hamilton's programme.

It has taken two years for the mathematical community to assimilate Perelman's ideas and expand his preprints into complete proofs. Very recently, an article [1] and a book [7] containing complete and mostly self-contained proofs of the Poincare

Date: August 14, 2006. 

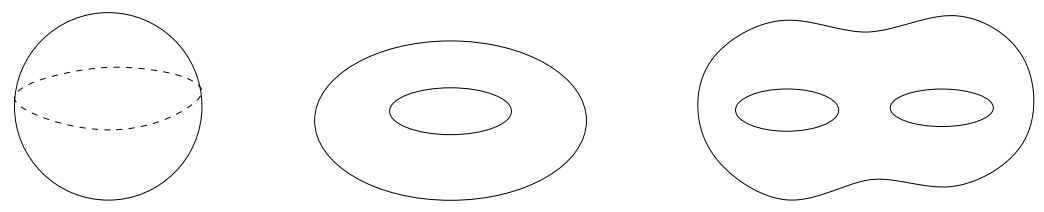

FIgURE 1. The first three orientable surfaces

conjecture have been posted. An earlier set of notes which filled in many details in Perelman's papers is [5].

In this article we attempt to give an exposition of Perelman's work and the mathematics that went into it.

Acknowledgements. It is our pleasure to thank Kalyan Mukherjea for several helpful comments that have considerably improved the exposition. We would also like to thank Basudeb Datta, Gautham Bharali and Joseph Samuel for helpful comments.

\section{Some notions of TOpology}

In this section, we informally formalise the Poincaré conjecture. To do this, we first need to introduce the higher-dimensional analogues of surfaces, namely smooth manifolds. For those in the know, we consider throughout diffeomorphism types of smooth manifolds as this suffices in dimension 3.

To introduce manifolds we take a closer look at surfaces. A surface in $\mathbb{R}^{3}$ is the set of zeroes of a smooth function $f(x, y, z)$ which is non-singular, i.e., for each point on the surface the gradient $\nabla f(x, y, z)$ of $f$ is non-zero. Basic examples of this are the plane $z=0$ and the sphere $x^{2}+y^{2}+z^{2}-1=0$.

In analogy with this, we can consider a subset $M \subset \mathbb{R}^{n}$ which is the set of zeroes of $n-k$ smooth functions $f_{1}, \ldots, f_{n-k}$ whose gradients $\nabla f_{i}$ are linearly independent for all points in $M$. Such a subset of $\mathbb{R}^{n}$ is a $k$-dimensional manifold or a $k$-manifold.

More generally, a set $M$ given as above may have several components. We consider each component of $M$ to be a $k$-manifold. For the rest of this article, by a $k$-manifold $M$ we mean a component of the subset $M \subset \mathbb{R}^{n}$ which is the set of zeroes of $n-k$ smooth functions $f_{1}, \ldots, f_{n-k}$ whose gradients $\nabla f_{i}$ are linearly independent for all points in $M .^{1}$

We say that two smooth $k$-dimensional manifolds $M$ and $N$ are diffeomorphic if there is a smooth one-to-one correspondence $f: M \rightarrow N$ between the points of the manifolds with a smooth inverse. The function $f$ is called a diffeomorphism.

We say that a manifold (defined as above) is closed or compact if it is contained in a bounded subset of $\mathbb{R}^{n}$.

In this language, the Poincaré conjecture can be stated as follows.

Conjecture (Poincaré). Any closed, simply-connected 3-manifold is diffeomorphic to the 3-dimensional sphere $S^{3}$.

For a brief history of the Poincaré conjecture, see [6].

A small region around any point in a surface can be given a pair of local coordinates. For example, away from the poles, the latitude and the longitude form coordinates for any small region on the sphere. Local coordinates correspond to

\footnotetext{
${ }^{1}$ This is equivalent to the usual definition by a theorem of Nash.
} 


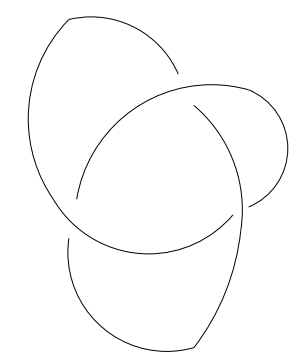

FiguRE 2. A knotted curve

making a map of a region of the surface on a piece of paper in such a way that objects that are close to each other on the surface remain close on the map. One cannot make a single such map of the whole surface, but it is easy to see that one can construct an atlas of such maps. Each map is usually called a chart.

Similarly, a small region around any point in a $k$-manifold $M$ can be given a system of $k$ local coordinates $x_{1}, \ldots x_{k}$. It is frequently convenient to study local properties of a manifold using these coordinates. These allow one to treat small regions of the manifold as subsets of Euclidean space, using a chart as in the case of surfaces. By using an atlas of such charts, one can study the whole manifold.

\section{Why the Poincaré CONJECTURe IS Difficult}

Both the plane and 3-dimensional space are simply-connected but with an important difference. If we take a closed, embedded curve in the plane (i.e., a curve which does not cross itself), it is the boundary of an embedded disc. However, an embedded curve in 3-dimensional space may be knotted (see figure 2). This means that as we deform a knotted curve to a point, along the way it must cross itself.

Thus, an embedded curve in a simply-connected 3-manifold $M$ may not bound an embedded disc. Furthermore, such a curve may not be contained in a ball $B$ in $M$. While embedded disks are useful in topology, immersed disks (i.e., disks that cross themselves) are not. It is this which makes the Poincaré conjecture difficult (in dimension 3).

The analogue of the Poincaré conjecture in dimensions 5 and above is easier than in dimension 3 for a related reason. Namely, any (2-dimensional) disc in a manifold of dimension at least 5 can be perturbed to an embedded disc, just as a curve in 3-dimensional space can be perturbed so that it does not cross itself.

What made Perelman's proof, and Hamilton's programme, possible was the work of Thurston in the 70's, where he proposed a kind of classification of 3-manifolds, the so called geometrization conjecture [11]. Thurston's geometrization conjecture had as a special case the Poincaré conjecture, but being a statement about all 3manifolds could be approached without using the hypothesis of simple-connectivity.

However most of the work on geometrization in the 1980's and 1990's was done by splitting into cases, so to prove the Poincaré conjecture one was still stuck with trying to use the simple-connectivity hypothesis. An exception to this was Hamilton's programme. Interestingly, Perelman found a nice way to use simple-connectivity within Hamilton's programme, which simplified his proof of the Poincaré conjecture (but not of the full geometrization conjecture). 


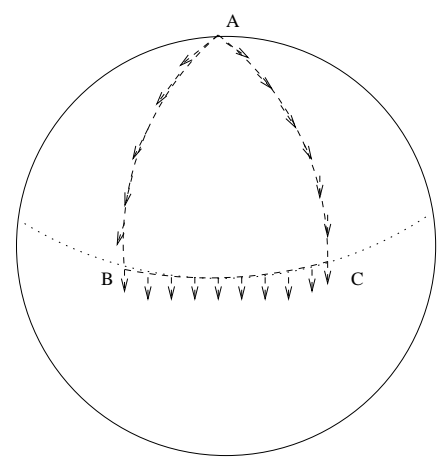

Figure 3. The holonomy on a sphere

To introduce Hamilton's approach we need to reformulate the Poincaré conjecture as a statement relating topology to Riemannian geometry, namely that a compact, simply-connected 3-manifold has an Einstein metric. To make sense of this we need some Riemannian geometry.

\section{Some Riemannian GEOMEtry}

3.1. Wind-vanes on spheres. Let us begin with a few thought experiments. Consider a frictionless wind-vane in vacuum. Assume that the wind-vane is initially stationary. Then if we rotate the base of the wind-vane, the arrow does not move (as there is no friction).

Now assume that the wind-vane is placed flat on a plane. Let us move the base of the wind-vane smoothly along a curve in the plane. Then the wind-vane points in a constant direction, i.e., the direction of the wind-vane gives parallel vectors along the curve. This gives a physical meaning to vectors at different points being parallel.

Something more interesting happens if we perform the same experiment on the surface of a sphere (see figure 3 ). Suppose the wind-vane is initially placed at the point $A$ which we take to be the north pole, and points in the direction of $B$ on the equator. Let $C$ be another point on the equator. We shall move the wind-vane along the longitude $A B$, then along the equator from $B$ to $C$ and finally back to $A$ along a longitude.

As we move along $A B$, the wind-vane is always pointing in the direction of motion. We can see this by symmetry - as the two sides of the longitude of a sphere look the same, the there is no reason for the wind-vane to turn right rather than left (or vice versa). When we reach $B$, the wind-vane is perpendicular to the equator, so that it is pointing due south. Now as we move along the equator to $C$ (by symmetry) it continues to point south. Hence when we reach $C$, we are pointing along the longitude through $C$ towards the south pole. Finally, as we move the wind-vane back along the longitude $A C$, it continues to point towards $C$.

Notice that something remarkable has happened. The wind-vane which was pointing towards $B$ is now pointing towards $C$. Thus if we look at parallel vectors along the loop $A B C A$, we end up with a different vector from the one we had started with. Thus parallel transport around a loop on a sphere leads to a rotation, in technical language the holonomy of the parallel transport. This happens because 
the sphere is curved, and the amount by which we rotate depends on the curvature. By contrast, in the plane the holonomy is always the identity, i.e., each vector is taken to itself.

We need some basic concepts before returning to parallel transport and curvature.

3.2. Intrinsic differential geometry. In intrinsic differential geometry, we study the geometry of a space $M$ in terms of measurements made on the space $M$. This began with the work of Gauss, who was involved in surveying large areas of land where one had to take into account the curvature of the earth. Even though the earth is embedded in 3-dimensional space, the measurements we make cannot take advantage of this.

Intrinsic differential geometry gained new importance because of the general theory of relativity, where one studies curved space-time. Thus, we have manifolds with distances on them that do not arise from an embedding in some $\mathbb{R}^{n}$. This depended on the higher-dimensional, and more sophisticated, version of intrinsic differential geometry developed by Riemann. Today, intrinsic differential geometry is generally referred to as Riemannian geometry.

To study Riemannian geometry, we need to understand the analogues of the usual geometric concepts from Euclidean geometry as well as the new subtleties encountered in the more general setting. Most of the new subtleties are captured by the curvature.

3.3. Tangent spaces. Let $M$ be a $k$-dimensional manifold in $\mathbb{R}^{n}$ and let $p \in M$ be a point. Consider all smooth curves $\gamma:(-1,1) \rightarrow M$ with $\gamma(0)=p$. The set of vectors $v=\gamma^{\prime}(0)$ for such curves $\gamma$ gives the tangent space $T_{p} M$. This is a vector space of dimension $k$ contained in $\mathbb{R}^{n}$. For example, the tangent space of a sphere with center the origin at a point $p$ on the sphere consists of vectors perpendicular to $p$.

If a particle moves smoothly in $M$ along the curve $\alpha(t)$, its velocity $V(t)=\alpha^{\prime}(t)$ is a vector tangent to $M$ at the point $\alpha(t)$, i.e., $V(t) \in T_{\alpha(t)} M$. In general if $\alpha(t)$ is a curve and $V(t)$ a vector in $T_{\alpha(t)} M$ for all $t$, then $V$ is said to be a (tangent) vector field along $\alpha$.

3.4. Riemannian metrics. If $\alpha:(a, b) \rightarrow M$ is a smooth curve then its length is given by $l(\alpha)=\int_{a}^{b}\left\|\alpha^{\prime}(t)\right\| d t$. Similarly, its energy, i.e., the total kinetic energy of a particle moving along the path $\alpha$, is given by $E(\alpha)=\int_{a}^{b}\left\|\alpha^{\prime}(t)\right\|^{2} d t$

Observe that these quantities are determined by the inner product (i.e., dot product) on the tangent spaces $T_{p}(M)$, and do not in any other way depend on how $M$ sits as a subset of $\mathbb{R}^{n}$. Thus, if we specify the inner product on $T_{p}(M)$ for each $p \in M$ (varying smoothly), then we can compute geometric quantities like lengths, energies, angles (using the cosine formula) etc..

A Riemannian metric $g$ on $M$ is an inner product specified on $T_{p} M$ for each $p \in M$. Thus, $g$ refers to a collection of inner products, one for each $T_{p} M$. We further require that $g$ varies smoothly in $M$. For a point $p \in M$ and vectors $V, W \in T_{p} M$, the inner product of $V$ and $W$ corresponding to the Riemannian metric $g$ is denoted $g(V, W)$.

A Riemannian manifold $(M, g)$ is a manifold $M$ with a Riemannian metric $g$ on it. Recall that near any point in $M$, a small region $U \subset M$ can be given a system 
of local coordinates $x_{1}, \ldots, x_{k}$. If we denote the corresponding coordinate vectors by $\hat{e}_{1}, \ldots, \hat{e}_{k}$, then for any point $p$ in $U$ the inner product on $T_{p} U$ is determined by the matrix $g_{i j}=g\left(\hat{e}_{i}, \hat{e}_{j}\right)$. This is a symmetric matrix.

The first examples of Riemannian manifolds are submanifolds $M \subset \mathbb{R}^{n}$, with the inner product on $T_{p} M$ the restriction of the usual inner product on $\mathbb{R}^{n}$. This metric is called the metric induced from $\mathbb{R}^{n}$.

A second important class of examples are product metrics. If $(M, g)$ and $(N, h)$ are Riemannian manifolds, we can define their product $(M \times N, g \oplus h)$. The points of $M \times N$ consist of pairs $(x, y)$, with $x \in M$ and $y \in N$. The tangent space $T_{(x, y)} M \times N$ of the product consists of pairs of vectors $(u, v)$ with $u \in T_{x} M$ and $v \in T_{y} N$. The inner product $(g \oplus h)$ is given by

$$
(g \oplus h)\left((u, v),\left(u^{\prime}, v^{\prime}\right)\right)=g\left(u, u^{\prime}\right)+h\left(v, v^{\prime}\right)
$$

We can identify the space of vectors of the form $(u, 0)$ (respectively $(0, v)$ with $T_{x} M$ (respectively $T_{y} N$ ).

3.5. Isometries. Two Riemannian manifolds $(M, g)$ and $(N, h)$ are said to be isometric if there is a diffeomorphism from $M$ to $N$ so that the length of any curve in $M$ is the same as that of the corresponding curve in $N$. In Riemannian geometry, we regard two isometric manifolds as the same.

3.6. Geodesics. Geodesics are the analogues of straight lines. A straight line segment is the shortest path between its endpoints. Furthermore, if a particle moves in space with no force acting on it, then it moves along a straight line at constant speed. It is this property we seek to generalise. To do this, however we need to consider not just minima (of the length or energy) but critical points, i.e. points at which the first derivative of the relevant function (length or energy in our case) vanishes. Elementary calculus tells us that all minima are critical points but the converse is not true.

According to Lagrangian mechanics, the path $\gamma(t)$ taken by a particle in the absence of any force is a critical point of the total kinetic energy of the particle along its path (among paths with fixed endpoints). As we have seen, this Energy can be defined on any Riemannian manifold. We define a geodesic to be a critical point of the energy among paths with fixed endpoints.

3.7. Extrinsic covariant derivatives. Newton's law says that in the absence of a force, a particle in Euclidean space has zero acceleration. However, if a particle moves in a sphere it cannot have zero acceleration (as a particle with zero acceleration moves along a straight line). Instead we may assume that the force, hence the acceleration, is perpendicular to the sphere. This would mean that the orthogonal projection of the acceleration on to the tangent space is zero.

In the Euclidean case, the acceleration is the derivative of the velocity. By the above, the natural replacement for this in the case of the induced metric on $M$ is to take the projection of the usual derivative. This is the covariant derivative in this special case. To distinguish this from the more general notion, we shall often refer to this as the extrinsic covariant derivative.

More precisely, let $\alpha(t)$ be a smooth curve in $M$ and let $V(t)$ be a smooth vector field along $\alpha$. We then define the covariant derivative $\frac{D V(t)}{d t}$ to be the orthogonal projection onto $T_{\alpha}(t)$ of the usual derivative $\frac{d V}{d t}$ of $V$. 
We say that a vector field $V$ as above is parallel if its covariant derivative is zero everywhere. A curve $\gamma(s)$ is a geodesic if and only if its velocity is parallel (in the case of an induced metric).

3.8. Intrinsic covariant derivatives. The extrinsic covariant derivative is unsatisfactory as it is not clear how to extend the definition to Riemannian metrics on $M$ different from the induced metric. There is an elegant way around this in much the same spirit as Euclid. In classical Euclidean geometry, one does not define lines, points or angles, but instead we have axioms relating these notions. One expects (though there are logical issues involved) that these axioms completely determine Euclidean geometry.

We proceed in the same way, guided by the extrinsic covariant derivatives. Namely we consider properties that we expect the covariant derivative to satisfy, and which are satisfied by the extrinsic covariant derivative. Once the axioms are correctly formulated, it is easy to show that these properties uniquely determine the covariant derivative.

The first two axioms, linearity and Leibniz rule, are the natural analogues of properties of the usual directional derivatives. The third, compatibility with the metric says that for a parallel vector field $V(s),\|V(s)\|$ is constant.

The forth axiom, torsion freeness, is equivalent to the statement that the acceleration $\frac{D \gamma^{\prime}(t)}{d t}$ of a geodesic $\gamma(t)$ is zero. This says that paths minimising energy satisfy Newton's law for motion in the absence of a force.

All the above properties are both geometrically and physically reasonable. As these uniquely determine the covariant derivative, we shall take them as the axioms that define the covariant derivative.

3.9. Curvature. The curvature of a surface measures the holonomy, which is the result of parallel transport around a loop. This can be readily quantified in terms of the covariant derivative.

Let $P \in M$ be a point and let $X, Y$ and $Z$ be tangent vectors at this point. Let $\alpha(s, t)$ be a smooth function with values in $M$ such that $\alpha(0,0)=P, \frac{d \alpha(0,0)}{d s}=X$ and $\frac{d \alpha(0,0)}{d t}=Y$. Extend $Z$ to a smooth vector field along $\alpha(s, t)$.

Define a vector $R(X, Y) Z$ by

$$
R(X, Y) Z=\frac{D}{d s} \frac{D}{d t} Z-\frac{D}{d t} \frac{D}{d s} Z
$$

The vector valued function $R(X, Y) Z$ is called the curvature tensor. Essentially, the holonomy around a parallelogram is the difference between performing parallel transport first along a horizontal side and then a vertical side and performing parallel transport first along a vertical side and then a horizontal side. As parallel vector fields are those whose covariant derivative is zero, it is not difficult to see that the curvature tensor (as defined above) measures the holonomy about a small parallelogram (more precisely, the appropriate limit of holonomies).

3.10. Gaussian, Sectional, Ricci, and Scalar curvatures. The curvature tensor is a complicated algebraic object. It is more useful to have numbers, whose sign and magnitude tell us about the geometry of the surface.

The simplest curvature is the Gaussian curvature, which is defined for a surface $(M, g)$ (i.e., a 2-dimensional Riemannian manifold). Let $p \in M$ be a point and let 
$X, Y \in T_{p} M$ be orthogonal unit vectors. Then we define $K(p)=g(R(X, Y) X, Y)$. This is independent of $X$ and $Y$.

In higher dimensions, if we take $X$ and $Y$ as above, we still get a curvature, but this now depends on the plane $\xi$ in $T_{p} M$ spanned by $X$ and $Y$ (in the case of surfaces, the plane is all of $\left.T_{p} M\right)$. This is called the sectional curvature $K(p, \xi)$ at $p$ along the plane $\xi$. We sometimes denote the sectional curvature by $K_{g}(x, \xi)$ to clarify what metric we consider.

Averaging all the sectional curvatures at a point gives the scalar curvature $R(p)$. There is an intermediate quantity, called the Ricci tensor which is very fundamental in our situation. This is defined by taking the appropriate trace as below.

Fix vectors $U$ and $V$ in $T_{p} M$, which need not be orthogonal or of unit length. Then $W \mapsto R(U, W) V$ is a linear transformation on $T_{p} M$. A basic result in Riemannian geometry says that the matrix of this linear transformation (with respect to an orthonormal basis for the inner product from $g$ ) is symmetric. We define $\operatorname{Ric}(U, V)$ to be the trace of this matrix.

Another basic property of the curvature tensor is that this is symmetric in $U$ and $V$, i.e., $\operatorname{Ric}(U, V)$ is a symmetric bilinear form. The somewhat unnatural definition is to exploit these symmetries. Recall that the Riemannian metric $g$ is also given in local coordinates by a symmetric matrix. The Ricci flow exploits this analogy.

We consider some examples.

(1) Euclidean space. This is just $\mathbb{R}^{n}$ with the usual inner product. In this case, all the sectional curvatures are zero. Hence so is the Ricci tensor and the scalar curvature.

(2) Sphere $S^{n}(r)$ of radius $r$ with the metric induced from $\mathbb{R}^{n+1}$. In this case, all sectional curvatures are equal to $r^{-2}, \operatorname{Ric}(U, V)=(n-1) r^{-2} g(U, V)$ and $R(p)=n(n-1) r^{-2}$ for any point $p$. Here $g(\cdot, \cdot)$ is (the restriction of) the standard inner product in $\mathbb{R}^{n}$

(3) There is an analogue of Example 2, called hyperbolic space, for which the sectional curvature is $-r^{-2}$. The underlying manifold can be taken to be $\mathbb{R}^{n}$. We will not describe the metric since we won't need it.

We have the following important converse of the above examples: Let $(M, g)$ be a simply-connected complete Riemannian manifold of constant sectional curvature $k$. Then $M$ is isometric to Euclidean space, the sphere of radius $\sqrt{1 / k}$ or hyperbolic space according as $k=0, k>0$ or $k<0$ respectively.

(4) A product Riemannian manifold $\left(M \times N, g=g_{1} \oplus g_{2}\right)$ : If $\xi$ is a plane in $T_{p}(M \times N)$ that is tangent to $M($ respectively $N)$, then $K(p, \xi)=K_{1}(\xi)$ (respectively $K_{2}(\xi)$ ). Here $K_{1}$ and $K_{2}$ denote the sectional curvatures with respect to $g_{1}$ and $g_{2}$. On the other hand, if $\xi$ is the span of a vector tangent to $M$ and one tangent to $N$, then $K(\xi)=0$

(5) As a special case of the above, consider a surface $M$ which is the product of two circles, possibly of different radii, with the product metric. Then the tangent plane at any point is spanned by a vector tangent to the first circle and one tangent to the second circle. Hence the sectional curvature of $M$ at any point is zero.

(6) Another example of a product metric that we need is that on $M=S^{2} \times \mathbb{R}$. In this case, the sectional curvature $K(x, \xi)$ is 1 if $\xi$ is the tangent plane of $S^{2}$ and 0 if $\xi$ contains the tangent space of $\mathbb{R}$. 
3.11. The Jacobi equation. We would like to relate the geometry of a manifold to its curvature. The best way to do this is through the Jacobi equation. We shall consider the case of surfaces and mention how this case generalises.

Consider a family of geodesics $\alpha_{u}(T)$ starting at $p$, all of which have unit speed. Let $\alpha(t)=\alpha_{0}(t)$ and let $N(t)$ be the unit vector at the point $\alpha(t)$ normal to $\alpha^{\prime}(t)$.

The Jacobi vector field is $J(t)=\left.\frac{\partial}{\partial u} \alpha_{u}(t)\right|_{u=0}$. In the case of surfaces, this is of the form $J(t)=f(t) N(t)$.

By differentiating the equation for a geodesic and using the definition of curvature, we can see that the Jacobi vector fields $J(t)=f(t) N(t)$ on surfaces satisfy the Jacobi equation

$$
\frac{d^{2} f}{d t^{2}}+K(\alpha(t)) f=0
$$

If the curvature $K$ is zero, then this says that $f$ is a linear function, as happens in the Euclidean plane. In particular, geodesics move apart at a constant rate. If $K=1$, the solutions to the equation are linear combinations of the trigonometric functions $\sin (t)$ and $\cos (t)$. Thus, geodesics bend towards each other and meet in a finite time. This happens, for instance, on the sphere. Finally, if $K=-1$, solutions are exponential functions, so geodesics diverge exponentially fast.

One can derive similar conclusions for positive or negative (not necessarily constant) curvature metrics, using the Sturm-Liouville theory of ordinary differential equations.

In higher dimensions, we can derive a similar Jacobi equation

$$
\frac{D^{2} J}{d t^{2}}+R(V, J) V=0
$$

where $V(t)=\alpha^{\prime}(t)$.

In case the sectional curvature is positive (respectively negative), i.e., is positive (respectively negative) at each point and in each plane at that point, we can reason as in the case of surfaces. One can still draw some conclusions in the case of positive Ricci curvature.

3.12. Manifolds with non-negative sectional curvature. By the Jacobi equation, if a Riemannian manifold has non-negative curvature, geodesics do not diverge faster than in Euclidean space. This has strong consequences for the geometry and topology of these manifolds. In particular, if a simply-connected 3-manifold $(M, g)$ has non-negative sectional curvature, it is diffeomorphic to one of $\mathbb{R}^{3}, S^{3}$ and $S^{2} \times \mathbb{R}$. Moreover, if $(M, g)$ is diffeomorphic to $S^{2} \times \mathbb{R}$ then $g$ is a product metric.

3.13. Scaling and curvature. Suppose $(M, g)$ is a Riemannian manifold and $c>$ 0 is a constant. Then the sectional curvature $K^{\prime}$ of the Riemannian manifold $(M, c g)$ is related to the sectional curvature $K$ of $(M, g)$ by

$$
K^{\prime}(p, \xi)=c^{-1} K(p, \xi)
$$

for every point $p \in M$ and every tangent plane $\xi \subset T_{p} M$ at that point.

Note that if $c$ is large, then $K^{\prime}$ is small. Hence, given a compact Riemannian manifold $(M, g)$ we can always choose $c$ large enough so that $(M, c g)$ has sectional curvatures lying between -1 and 1 . 


\section{Einstein metrics And the Poincaré COnjecture}

An Einstein metric is a metric of constant Ricci curvature. More precisely, an Einstein metric with constant curvature $a$ is a metric that satisfies, for all $p \in M$ and $U, V \in T_{p} M$, the equation

$$
\operatorname{Ric}(U, V)=a g(U, V) .
$$

These can also be characterised as being the critical points among metrics with fixed volume of the Einstein-Hilbert action in general relativity.

To relate Einstein metrics to the Poincare conjecture, one notes that an Einstein metric $g$ on a 3-manifold necessarily has constant sectional curvature (in all dimensions metrics of constant sectional curvature are Einstein metrics). Hence, by 3.10, one concludes that if $(M, g)$ is closed, simply-connected and Einstein, then $(M, g)$ is isometric to $S^{3}$ with a round metric. Note that we can rule out Euclidean and Hyperbolic space since they are not closed. In particular, $M$ is diffeomorphic to $S^{3}$.

Hence the Poincaré conjecture can be formulated as saying that any closed, simply-connected 3-manifold has an Einstein metric. More generally, Thurston's geometrisation conjecture says that every closed 3-manifold can be decomposed into pieces in some specified way so that each piece admits a locally homogeneous metric, a concept more general than that of a metric with constant sectional curvature.

\section{HAMilton's RiCCI FLOW}

In the 1980's and 1990's Hamilton built a programme to prove geometrisation, beginning with a paper [3] where he showed that if a 3-manifold has a metric with positive Ricci curvature then it has an Einstein metric. By positive Ricci curvature we mean that if $p \in M$ and $U \in T_{p} M$ is non-zero, then $\operatorname{Ric}(U, U)>0$.

Hamilton's approach was to start with a given metric $g$ and consider the 1parameter family of Riemannian metrics $g(t)$ satisfying the Ricci flow equation

$$
\frac{\partial g}{\partial t}=-2 R i c(t), \quad g(0)=g
$$

where $\operatorname{Ric}(t)$ is the Ricci curvature of the metric $g(t)$.

To get a feeling for the analytical properties of this equation, we first consider the simpler case of the heat equation which governs the diffusion of heat in an isolated body. The heat equation is

$$
\frac{\partial u}{\partial t}=\Delta u
$$

The temperature in an isolated body becomes uniform as time progresses; one hopes similarly that the Ricci flow makes the Ricci curvature uniform. Further, the minimum temperature of the isolated body increases (and the maximum temperature decreases) with time. This latter property is called a maximum principle.

To see the relation of the Ricci flow with the heat equation, we use special local coordinates called harmonic coordinates (i.e., coordinates $\left\{x_{i}\right\}$ such that the functions $x_{i}$ are harmonic). We can find such coordinates around any point in a Riemannian manifold $M$. In these coordinates we have

$$
R i c_{i j}=-\frac{1}{2} \triangle g_{i j}+Q(g, \partial g)
$$


where $Q$ is an expression involving $g$ and the first partial derivatives of $g$ and $R_{i j}=\operatorname{Ric}\left(\hat{e}_{i}, \hat{e}_{j}\right)$.

Hence the Ricci flow resembles the heat flow $\frac{\partial g_{i j}}{\partial t}=\triangle g_{i j}$ leading to the hope that the Ricci curvature becomes uniform as time progresses. However, there is an extra term $Q(g, \partial g)$ of lower order. Such a term is called the reaction term and equations of this form are known as reaction-diffusion equation. In order to understand such an equation, one needs to understand both the nature of the reaction term and conditions that govern whether the reaction or the diffusion terms dominate.

Let us consider some examples: If $g$ is the induced metric on the sphere $S^{3}$ of radius 1 , then $g(t)=(1-4 t) g$ is the solution to (1). Note that the radius of $\left(S^{3}, g(t)\right)$ is $\sqrt{1-4 t}$ and the sectional curvatures are $\frac{1}{1-4 t}$. As $t \rightarrow \frac{1}{4}$, these curvatures blow-up.

More generally, if $g(t)$ is an Einstein metric the Ricci flow simply rescales the metric. In fact, if Ric $=a g$, then $g(t)=(1-2 a t) g$ satisfies (1). Note that $(M, g(t))$ shrinks, expands or remains stationary depending on whether $a>0, a<0$ or $a=0$.

On the other hand, if the metric is fixed up to rescaling by the Ricci flow then it is an Einstein metric.

We need to consider a more general class of solutions $g(t)$, called the Ricci solitons for which the manifolds $(M, g(t))$ are isometric up to scaling. More precisely, a Ricci soliton is a solution $g(t)$ so that for each time $t$ there is a constant $c(t)$ such that $(M, g(t))$ and $(M, c(t) g(0))$ are isometric.

Let $\left(M_{1} \times M_{2}, g_{1} \oplus g_{2}\right)$ be a product Riemannian manifold. Then the Ricci flow beginning at $g_{1} \oplus g_{2}$ is of the form $g(t)=g_{1}(t) \oplus g_{2}(t)$, where $g_{1}(t)$ and $g_{2}(t)$ are the flows on $M_{1}$ and $M_{2}$ beginning with $g_{1}$ and $g_{2}$. Ricci flow preserves product structure. In particular, the flow beginning with the standard product metric $g_{0} \oplus g_{1}$ on $S^{2} \times \mathbb{R}$ is $g(t)=(1-2 t) g_{0} \oplus g_{1}$, i.e., the $S^{2}$ shrinks while the $\mathbb{R}$ direction does not change. This example is crucial for understanding regions of high curvature along Ricci flow.

We now consider some analytical properties of the Ricci flow. One of the first results proved by Hamilton was that, given any initial metric $g(0)$ on a smooth manifold $M$, the Ricci flow equation has a solution on some time interval $[0, \epsilon)$. Furthermore, this solution is unique. It follows that a solution to the equation with initial metric $g(0)$ exists on some maximal interval $[0, T)$, with $T$ either finite or infinite and is unique on this interval.

Further, if $T$ is finite the curvature becomes very large as we approach $T$. More precisely, there is a sequence of points $x_{i}$, times $t_{i} \in[0, T)$ with $t_{i} \rightarrow T$ and planes $\xi_{i} \in T_{x_{i}} M$ such that the sectional curvatures $K_{g\left(t_{i}\right)}\left(x_{i}, \xi_{i}\right)$ of the metric $g\left(t_{i}\right)$ at $x_{i}$ for the plane $\xi_{i}$ goes to $\pm \infty$. Often we choose $x_{i}, \xi_{i}$ and $t_{i}$ with maximal curvature in the sense that $\left|K_{g\left(t_{i}\right)}\left(x_{i}, \xi_{i}\right)\right| \geq\left|K_{g\left(t^{\prime}\right)}(y, \zeta)\right|$ for all times $t^{\prime} \in\left[0, t_{i}\right]$ and all $y \in M$, $\zeta \in T_{y} M$.

The main idea of Hamilton's programme is to evolve an arbitrary initial metric on a closed simply-connected 3-manifold along the Ricci flow and hope that the resulting metric converges, up to rescaling, to an Einstein metric. Hamilton showed that this does happen when $g$ has positive Ricci curvature.

It is convenient to analyse separately the cases where the maximal interval of existence $[0, T)$ is finite and infinite. It turns out, as we explain later, that if the 
manifold is simply-connected, then this time-interval is finite. In particular, the curvature blows-up in finite time on certain parts of the manifold.

The central issue in Hamilton's programme was to understand, topologically and geometrically, the parts of the manifold where curvature blows-up along the Ricci flow.

\section{PinCHING AND ZOOM}

The first major steps in understanding the geometry near points of large sectional curvature were due to Hamilton and Ivey, using maximum principles.

In the simple case of a heat equation we have a maximum principle that says that if the temperature is initially greater than a constant $\alpha$ at all points in the manifold, then this continues to hold for all subsequent times. In the case of the Ricci flow, we have a similar maximum principle for the scalar curvature. This is because the reaction term for the evolution of the scalar curvature is positive. As a consequence, the scalar curvature evolving along the Ricci flow is larger than the solution to the heat equation with the same initial conditions. In particular, we obtain the important conclusion that scalar curvature $R$ is bounded below along the Ricci flow.

Hamilton also developed a maximum principle for tensors which enabled him to prove that if $\operatorname{Ric}(g)>0$, then $\operatorname{Ric}(g(t))>0$ where $g(t)$ is the solution to Ricci flow with $g(0)=g$. More generally, starting with an arbitrary metric, Hamilton and Ivey independently obtained an inequality for the curvature using this maximum principle. All these maximum principles amount to showing and using positivity properties of the reaction term.

Now, suppose $x_{i}, \xi_{i}$ and $t_{i}$ are as in the previous section, It follows from the results of Hamilton and Hamilton-Ivey that $R\left(x_{i}\right) \rightarrow \infty$. This is very remarkable it says that if some sectional curvatures become large in magnitude, the average of the sectional curvatures also becomes large. Furthermore, if $R(x)$ is large, (at some time $t$ ) the pinching estimate says that if the sectional curvature of some plane is negative at $x$, its magnitude is small compared to $R(x)$.

The precise statement of the Hamilton-Ivey result is the following: There is a non-increasing function $\phi: \mathbb{R} \rightarrow \mathbb{R}$, such that $\lim _{y \rightarrow \infty} \phi(y)=0$, and a constant $C$ such that

$$
K(x, t) \geq-\phi(R(x, t))|R(x, t)|+C,
$$

for any sectional curvature $K(x, t)$. Since $R$ is bounded below, this inequality leads to the conclusions mentioned above.

This can be exploited using a classical technique in partial-differential equations called blow-up analysis. Suppose we choose $x_{i}$ and $\xi_{i}$ with maximal curvature as in the previous section. We rescale the metric $g\left(t_{i}\right)$ by $\lambda_{i}=\left|K_{g\left(t_{i}\right)}\left(x_{i}, \xi_{i}\right)\right|$. Then all sectional curvatures of $\left(M, \lambda_{i} g\left(t_{i}\right)\right)$ lie between 1 and -1 and some sectional curvature at $x_{i}$ is equal to 1 . Recalling from 3.13 the scaling properties of curvature, it follows from the Hamilton-Ivey inequality that if the sectional curvature of $\lambda_{i} g\left(t_{i}\right)$ is negative somewhere, it has very small magnitude. Thus, the metric is almost non-negatively curved.

To exploit this further, one needs to consider limits of the above metrics. 
Figure 4. An example of convergence

\section{Convergence of Riemannian manifolds}

We have seen above that it is important to understand limits of Riemannian metrics. First we recall a simpler situation.

Consider a bounded sequence $\left\{x_{n}\right\}$ of points on the real line or in the plane. Such a sequence may not be convergent, as it may oscillate (for example consider the sequence $x_{n}=(-1)^{n}+\frac{1}{n}$ on the real line). However, some subsequence of $x_{n}$ converges. A point to which some subsequence of $x_{n}$ converges is called a limit point (in our example 1 and -1 are the limit points as they are the limits of odd and even terms). One can deduce that, for large $n, x_{n}$ is close to one of the limit points (e.g., $(-1)^{n}+\frac{1}{n}$ is close to 1 or -1 ). Finally, note that if the sequence is not bounded, it may have no limit points.

A space $X$ is said to be compact if every sequence in the set has a limit point in $X$. Manifolds that are closed in terms of our earlier definition are compact.

We consider now the analogous situation where we have a sequence of Riemannian manifolds. We would like an analogue of the boundedness condition on points in the plane to ensure compactness. This allows us to understand the properties of a sequence of manifolds by understanding the properties of the limit points (which are Riemannian manifolds).

Firstly, we need to specify what we mean by convergence of Riemannian manifolds. Equivalently we need to say when the distance between two manifolds is small (say less than $\epsilon$ ). Recall that two Riemannian manifolds $(M, g)$ and $(N, h)$ are isometric (i.e., equal) if there is a diffeomorphism $f: M \rightarrow N$ so that for every curve $\alpha$ in $M$, the length of $\alpha$ is equal to that of the corresponding curve $f \circ \alpha$ in $N$. In the case of compact manifolds, we say that the distance between $M$ and $N$ is less than $\epsilon$ if there is a diffeomorphism $f: M \rightarrow N$ so that for every curve $\alpha$ in $M$, the ratio of the length of $\alpha$ to that of $f \circ \alpha$ is between $1-\epsilon$ and $1+\epsilon$ (i.e., close to 1$)$. We call a map $f$ as above an $\epsilon$-almost isometry.

In order to study convergence of non-compact Riemannian manifolds, we need to specify basepoints. By $(M, p)$ we henceforth mean a manifold $M$ with the basepoint $p \in M$. Given two such manifolds $(M, p)$ and $(N, q)$ with basepoints, we say that the distance between them is less than $\epsilon$ if there is an $\epsilon$-almost isometry $f: B_{M} \rightarrow B_{N}$ between the ball $B_{M}$ of radius $1 / \epsilon$ around $p$ in $M$ and the ball $B_{N}$ of radius $1 / \epsilon$ around $q$.

In order to assert that a given sequence of Riemannian manifolds has a convergent subsequence, it turns out that we need two conditions - bounded curvature and noncollapse. The first simply says that there is a bound $C$ such that the magnitude of the sectional curvatures is bounded above by $C$ for all planes at all points in all the manifolds $M_{i}$.

The second condition is required to ensure that we do not collapse to a lower dimensional space. The non-collapsing condition is that the volumes of all the balls of radius 1 in all the manifolds $M_{i}$ are bounded below by some positive number $V_{0}$.

Let us consider some examples to illustrate these concepts. In the first, we let $M_{i}=S^{2}, p_{i}=p$ and $g_{i}=i g$, where $g$ is the usual round metric on the two-sphere 

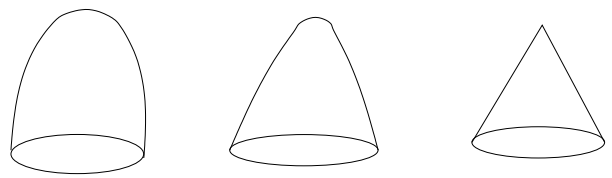

Figure 5. A sequence without bounded curvature with the limit singular
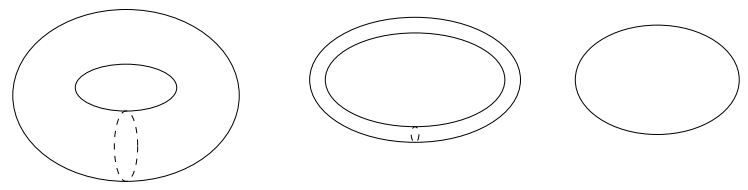

FiguRE 6. An example of collapsing
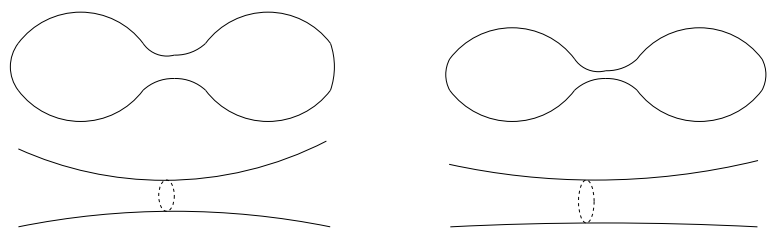

Limiting manifold

FiguRE 7. Sequences of manifolds and their blow-up limits

$S^{2}$ and $p$ is a fixed point in $S^{2}$. In this case, $\left(M_{i}, g_{i}, p_{i}\right)$ converges to Euclidean plane with the flat metric. Another example is illustrated in Figure 4.

For our next example, let $M_{i}=S^{1} \times S^{1}$ be the 2-torus, $g_{i}=i^{-1} g_{0} \oplus g_{0}$ and $p_{i}=(p, q)$, where $g_{0}$ is the usual metric on the circle. Observe that $\left(M_{i}, g_{i}\right)$ is the torus with the product metric obtained by viewing the torus as a product of a circle of radius $1 / i$ with a circle of radius 1 .

In this case the sectional curvature of $\left(M_{i}, g_{i}\right)$ is zero for any $i$. On the other hand, the limit of this sequence of metrics is the degenerate metric $0 \oplus g_{0}$. Hence the limit of the Riemannian manifolds (in the appropriate sense) is a circle (see Figure 6), which has a lower dimension than the spaces we considered. Note that the volume of any ball of radius 1 in $\left(M_{i}, g_{i}\right)$ is proportional to $i^{-1}$, hence noncollapse condition is violated.

One of the major results of Perelman was that in the situation of the blow-up limit of the previous section, i.e., when the metrics $g_{i}$ arise as rescalings at certain times along the Ricci flow, the non-collapsing condition holds.

Suppose we choose $x_{i}$ to have maximal curvatures. If we rescale so that the curvature at $x_{i}$ is 1 , then the curvature everywhere is at most 1 . Also $\left(M, c_{i} g\left(t_{i}\right)\right)$ satisfies the non-collapsing condition by Perelman. Hence, by the above we get limiting Riemannian manifolds. Our rescaled manifolds are close to these limiting manifolds which have, by the Hamilton-Ivey pinching estimate, non-negative sectional curvature.

\section{PERELMAN's CANONICAL NEIGHBOURHOOdS}

Let us assume that we have a Ricci flow on a simply-connected, closed 3-manifold with the curvature of the flow blowing up in finite time $T$. The results of the 
previous section can be summarized as follows: Consider a sequence of times $t_{i}$ increasing to $T$ and points $x_{i}$ of maximal curvature $\lambda_{i}$ (at time $t_{i}$ ). Rescaling $g\left(t_{i}\right)$ by $\lambda_{i}$, we have seen that a subsequence of $\left(M, \lambda_{i} g\left(t_{i}\right)\right)$ converges to a non-negatively curved manifold. We recall that the only simply-connected non-negatively curved manifolds in dimension 3 are $\mathbb{R}^{3}, S^{3}$ and $S^{2} \times \mathbb{R}$, with a product metric in the third case. Using this and other special properties of the limit, it can be proved that the limiting manifold is "standard". Hence, by definition of the limit manifold, it follows that small neighbourhoods of the points $x_{i}$ are close to being "standard". However, this procedure does not work if we want to understand points with high curvature which are not the maximal curvature points. The problem is that rescaling with respect to these points does not give metrics with curvature bounded independent of $i$.

A surprising and remarkable result of Perelman, which overcomes this difficulty and can be considered to be one of the central results in his proofs is the canonical neighbourhood theorem. This says that any point of high scalar curvature has a canonical neighbourhood which is a Riemannian manifold with metric very similar to a spherical space form or a neck $S^{2} \times I$ or is a so called $\epsilon$-cap. If $M$ is simply-connected, the spherical space form must be a sphere and the $\epsilon$-cap must be diffeomorphic to a 3-dimensional ball.

This result is surprising in many ways. Normally, by the kind of rescaling argument sketched above, we can study a neighbourhood of a point of maximal curvature. However, one expects that near points of high (but not maximal) curvature, there are nearby points where the curvature is much higher.

Thus, in general one expects the structure of curvature to be like a fractal, with peaks of many scales. Further, even very small regions of high curvature may have non-trivial topology and geometry. This is because, for any closed manifold, we can rescale the metric to make the diameter very small, at the cost of making curvature high.

One can view this in the more general context of complex systems arising from reaction-diffusion equations, of which the Ricci flow is a typical case. Typically, one gets a variety of behaviour at various scales emerging from the system. Hence it may be of considerable value to understand why this does not happen in the case of Ricci flow in dimension 3.

The proof of the canonical neighbourhood theorem is a subtle argument which again involves taking limits of Riemannian manifolds, among other things. A rough sketch is given in Section 12 .

\section{OutLine OF THE PROOF}

We are now in a position to outline the proof of the Poincaré conjecture. Consider a simply-connected 3-manifold $M$ with a Riemannian metric on it. We evolve this along the Ricci flow for the largest interval $[0, T)$ on which the flow is defined.

As a special case of the finite extinction property, whose proof we sketch below, $T$ must be finite. Thus, for some finite $T$, as we approach $T$ the scalar curvature goes to infinity on a subset of $M$.

Consider the subset $\Omega_{\rho}$ of $M$ where the curvature is bounded by a large number $\rho$ for all $t \in[0, T)$ i.e., let $\Omega_{\rho}=\{x \in M \mid R(x, t) \leq \rho$ for all $t\}$. We choose $\rho$ large enough that points of curvature greater than $\rho$ have a canonical neighbourhood. 


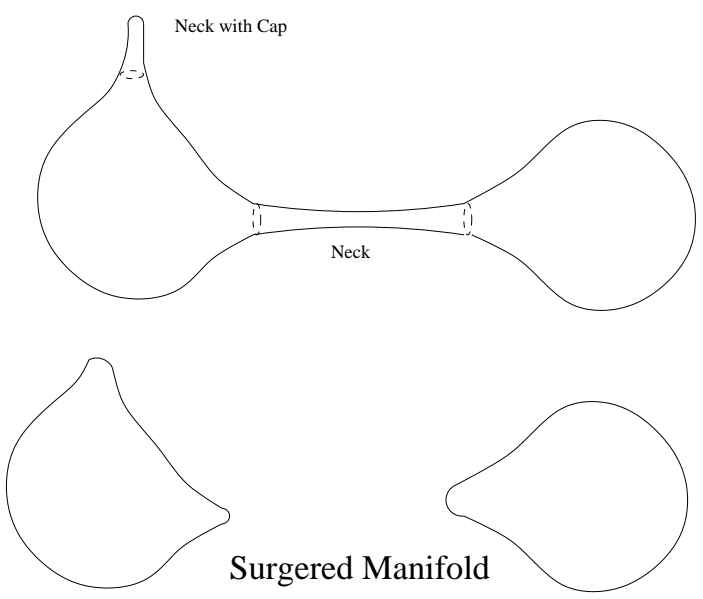

FIGURE 8. Surgery

For a time $t$ close to $T$, the canonical neighbourhood theorem holds for the complement $N$ of the interior of $\Omega_{\rho}$. Thus, every point in this complement has a neighbourhood that is a neck, a cap or diffeomorphic to a sphere. Putting these neighbourhoods together, we get either a sphere or a manifold diffeomorphic to $S^{2} \times[-1,1]$ (which is a union of several necks) which may have a cap attached at one or both ends. Topologically in each of these cases we obtain a sphere, a ball, or $S^{2} \times[-1,1]$. It follows in particular that the boundary of $\Omega_{\rho}$ consists of 2 -spheres.

If $\Omega_{\rho}$ is empty however large we choose $\rho$, in other words if the curvature blowsup on the entire manifold $M$, then the above implies that $M$ is diffeomorphic to $S^{3}$ and would be done.

Otherwise, we discard the set $\operatorname{int}(N)=M-\Omega_{\rho}$ and we attach balls to each of the boundary spheres of $\Omega_{\rho}$ to get a Riemannian manifold. This operation is called surgery.

Now we continue to evolve the manifold, which now has several components, by the Ricci flow. Once more, we evolve the metric along the Ricci flow till the curvature blows up, and repeat the above surgery procedure. Note that as we flow a component in the above process, either the curvature blows up everywhere or necks are formed. In the former case, we know that the component is a sphere. Such components are said to become extinct, and we continue the Ricci flow without them. In this case, the surgery is viewed as deleting the component.

Thus, we can inductively define the process called Ricci flow with surgery (see Figure 8). We need technical results that say that all the properties that we have for the ordinary Ricci flow hold for Ricci flow with surgery. We also need a result saying that in any finite time interval only finitely many surgeries are required to show that Ricci flow with surgery can be defined for all positive times.

A result of Perelman (for which a simpler and more elegant proof was provided by Colding and Minicozzi [2]) says that if the manifold $M$ is simply-connected, then all the components of the Ricci flow with surgery become extinct in finite time. This is proved by considering a geometric quantity called the waist and showing that it goes to zero in finite time. 
Thus, the above process becomes extinct in finite time. If we view this process backwards from the extinction time, we see that either spheres are created (the opposite of extinction) or two components are connected by a tube (the opposite of surgery). It is easy to see that the result after finitely many such steps is a collection of spheres. Thus, the manifold $M$ we started with must have been a sphere.

In the rest of the paper we sketch some of the techniques involved in proving the results mentioned.

\section{Perelman's Entropy functional}

Up to the work of Perelman, it was not even known that there were no non-trivial periodic orbits for the Ricci flow, i.e., where the initial metric is not a soliton, but after flowing according to the Ricci flow for a finite time, one ends up with the metric isometric to the one started with up to scaling. Periodic orbits can occur for arbitrary smooth flows, but not gradient flows of a function as the function must decrease along the gradient flow.

One of the first constructions of Perelman was that of an entropy functional, with the Ricci flow being the gradient flow of the negative of this entropy up to change of coordinates (i.e., up to a diffeomorphism). This was used to rule out periodic orbits for the Ricci flow. The entropy and its extensions were used to prove many results, including the non-collapsing results.

To construct the entropy, one starts with a manifold $M$ with a fixed volume element (technically, a smooth measure) $d V_{0}$ on it. In local coordinates, this means that we are given a positive density function $\rho_{0}$ so that the volume of a region is defined to be the integral of $\rho_{0}$ on the region.

Any Riemannian metric $g$ has a volume element $d V_{g}$ associated with it, with density in local coordinates $\operatorname{det}\left(g_{i j}\right)$. We can find a function $f$ such that $d V=$ $e^{f} d V_{0}$. Given this, Perelman defines an entropy functional by

$$
\int R+\|\nabla f\|^{2}
$$

As mentioned above, the Ricci flow is, up to change of coordinates, the gradient flow of the negative of the entropy. Thus entropy increases along the Ricci flow, ruling out periodic orbits. Note that as we have to rule out periodic orbits up to scaling, there are additional arguments needed.

Further conclusions can be obtained by using the freedom in choosing the measure $d V_{0}$. For instance, we take $d V_{0}$ to be concentrated near a point $x$ in $M$. If $(M, g(t))$, rescaled to make the curvature bounded at $x$, collapses near $x$, then one can show that the entropy must go to zero. But this contradicts the result that the entropy is increasing, proving non-collapsing.

There is plenty of speculation as to the meaning of entropy, but we confine ourselves to quoting some perceptive remarks of Mike Anderson. Observe that there are Ricci flows along which the geometry of the manifold does not change, namely, the Ricci solitons. Further, for so called gradient Ricci solitons, the metric along the Ricci flow is fixed up to scaling and volume preserving change of coordinates. Thus, the entropy must be constant for such flows, i.e., these must be critical points for the entropy. A calculation shows that the the critical points of the above functional are precisely the gradient Ricci solitons. 


\section{Parabolic Rescaling AND ASYMPtotic SOlitons}

Consider now the situation where the curvature blows up at time $T$. We have seen that by rescaling by the maximal scalar curvature we can get limiting manifolds of positive curvature. One can do better than this - by rescaling time as well, we get a new solution to the Ricci flow equation on a large interval. By shifting in time, we can take this to be an interval of the form $[-A, 0]$, with $A$ large. This process is called parabolic rescaling.

More precisely, let $g(t)$ be a Ricci flow for $t \in[0, T)$. Given a scaling factor $\lambda>0$ and a time $\tau \in[0, T)$, we can define a new Ricci flow on the interval $[-\lambda \tau, \lambda(T-\tau)]$ by

$$
\tilde{g}(t)=\lambda g\left(\tau+\frac{t}{\lambda}\right)
$$

For the rescalings that we consider, $\lambda$ is the maximum of sectional curvature at time $\tau$ and $[0, T)$ will be the maximal interval of definition of the flow. Since $T<\infty$, we know that $\lambda \rightarrow \infty$.

Similar to the theorem guaranteeing the existence of limits of Riemannian manifolds, Hamilton proved a convergence theorem for manifolds with Ricci flows. In particular, we can consider limit points of the sequence of parabolically rescaled flows. These limits will then be what Perelman calls $\kappa$-solutions, i.e., solutions to the Ricci flow equation defined on $(-\infty, 0]$ of non-negative curvature which satisfy a non-collapsing condition.

In order to understand $\kappa$-solutions $(\mathcal{M}, h(t))$, Pereleman showed that we can associate an asymptotic soliton to such a solution. This is done as follows: Take an appropriate sequence of points $q_{k}$ and times $t_{k} \rightarrow-\infty$ and parabolically rescale $(\mathcal{M}, h(t))$ by $\lambda=t_{k}^{-1}$. These rescaled flows can be shown to converge to a soliton with special properties. Perelman then proved that the only such solitons are the standard flow on $S^{2} \times \mathbb{R}$ or a quotient of $S^{3}$ using non-negativity of curvature as well as properties deduced by an analysis of the entropy functional. This classification of asymptotic solitons leads to the required understanding of $\kappa$-solutions.

As a consequence, one obtains a canonical neighbourhood theorem for $\kappa$-solutions, i.e., one obtains a neighbourhood $U$ of $x$ and a time interval $\left(t_{1}, t_{2}\right)$ such that the Ricci flow on $U \times\left(t_{1}, t_{2}\right)$ is close to the usual flow on $S^{2} \times \mathbb{R}$ or $S^{3}$ or an $\epsilon$-cap.

Further, Perelman obtains an important technical result which gives a control on the oscillation of the scalar curvature for such canonical neighbourhoods. This plays a crucial role in proving the canonical neighbourhood theorem for points of high (but not maximal) curvature.

\section{Points of high scalar CuRvature}

For points of maximal scalar curvature, we can parabolically rescale to get a limiting $\kappa$-solution. By the above, these points have canonical neighbourhoods.

To proceed further, i.e., to deal with points of high curvature which may not be maximal curvature points, Perelman uses an ingenious inductive argument. A point is said to be good if it satisfies the canonical neighbourhood theorem. Perelman considers a sequence of 'maximally bad points' $y_{i}$, more precisely, bad points $y_{i}$ with scalar curvature $R_{i}$ so that any point with scalar curvature at least $2 R_{i}$ a good point. Then he considers a ball of some fixed radius $\rho$ in the metric $g_{i}$ rescaled so that the scalar curvature at $y_{i}$ becomes 1 . 
As $y_{i}$ is in general not a point of maximal curvature, there may be points where the curvature is larger than 1 in the rescaled metric. However, Perelman gives an elegant argument to show that the curvature is bounded in balls of fixed size $\rho$ (with respect to $g_{i}$ ) centered at $y_{i}$. Observe that any bad point automatically has curvature bounded by 2 , while the curvature in the neighbourhood of a good point does not oscillate by much, as remarked at the the end of the last section. So if $\rho$ is small enough it is easy to conclude that neither good points nor bad points have curvature above 4 in the rescaled metric.

As we have a bound on the curvature, one can consider the limit of the Riemannian metrics on some ball of size $\rho$. Finally, Perelman considers the largest such $\rho$ and shows that it must be infinite, i.e., we have a parabolic limit as with points of maximal curvature, allowing one to prove the canonical neighbourhood theorem. This is a geometric argument involving the theory of Alexandrov spaces with curvature bounded below. We shall not enter into the details but confine ourself to the remark that the result used there is one which rules out behaviour at many scales.

\section{Concluding Remarks}

The value of a mathematical theorem in Science and Engineering often lies not just in its statement but in the ideas that are developed in the course of proving the theorem. In this respect, Perelman's (and Hamilton's) work is very rich in ideas which, when digested, may have consequences in a wide range of subjects outside mathematics.

A recent instance of this is the phenomenon of bubbling or energy concentration, first discovered while studying minimal surfaces. This has now become the heart of non-linear analysis with an enormous range of applications.

In the case of Perelman's work and the Hamilton programme, there are at least two sets of ideas which may be fundamental elsewhere. The first set are those deduced from Hamilton's maximal principles and Perelman's entropy functional. These are true for the Ricci flow in all dimensions, and perhaps for a wide range of systems.

The second set of ideas which involve Perelman's constructions together with non-negative curvature show that the Ricci flow in dimension three does not show certain kinds of complex behaviour. As is well known, the non-negativity of the curvature is a dynamical property amounting to saying that the geodesic flow is not chaotic (i.e., does not depend sensitively on initial conditions). One may speculate that the ideas that went into studying the Ricci flow would give some insight into when a system exhibits complex behaviour.

\section{REFERENCES}

[1] H.-D. Cao, X.-P. Zhu A Complete Proof of the Poincaré and Geometrization Conjectures Application of the Hamilton-Perelman Theory of the Ricci Flow, Asian Journal of Mathematics, 10 (2006), no. 2, 185-492.

[2] T. H. Colding, W. P. Minicozzi II: Estimates for the extinction time for the Ricci flow on certain 3-manifolds and a question of Perelman, Journal of American Mathematical Society 18 (2005), no. 3, 561-569.

[3] R. S. Hamilton Three-manifolds with positive Ricci curvature, J. Differential Geom. 17 (1982), no. 2, 255-306.

[4] R. S. Hamilton The formation of singularities in the Ricci flow, Surveys in differential geometry, Vol. II (Cambridge, MA, 1993), 7-136, Internat. Press, Cambridge, MA, 1995.

[5] B. Kleiner, J. Lott Notes on Perelman's papers, math.DG/0605667. 
[6] J. Milnor Towards the Poincaré conjecture and the classification of 3-manifolds, Notices Amer. Math. Soc. 50 (2003), no. 10, 1226-1233.

[7] J. Morgan, G. Tian Ricci flow and the Poincaré conjecture, math.DG/0605667.

[8] G. Perelman The entropy formula for the Ricci flow and its geometric application, math.DG/0211159.

[9] G. Perelman Ricci flow with surgery on three-manifolds, math.DG/0211159.

[10] G. Perelman Finite extinction time for the solutions to the Ricci flow on certain threemanifolds, math.DG/0211159.

[11] W. P. Thurston Three-dimensional manifolds, Kleinian groups and hyperbolic geometry, Bull. Amer. Math. Soc. (N.S.) 6 (1982), no. 3, 357-381.

Department of Mathematics, Indian Institute of Science, Bangalore 560012

E-mail address: gadgil@math.iisc.ernet.in

E-mail address: harish@math.iisc.ernet.in 